\title{
47. EL DESARROLLO DE LA CONSTITUCIÓN AUSTRÍACA EN LOS ÚLTIMOS DOS DECENIOS: REFORMA DE LAS INSTITUCIONES
}

\author{
HEINZ SCHÄFFER \\ Catedrático de Derecho Público \\ Universidad de Viena
}


SUMARIO

INTRODUCCIÓN.-I. LAS MÁS IMPORTANTES MODIFICACIONES CONSTITUCIONALES DESDE 1970. A MODO DE BOSQUEJO.-A. Las modificaciones más importantes en la estructura constitucional. B. Las tendencias principales del desarrollo de la Constitución hasta 1986. Reforma de las instituciones. C. Ideas y tendencias de reforma de la época 1970 a 1986. II. EL ÚLTIMO PERIODO DE 1986-1989 (HASTA HOY).- A. Retorno al modelo gubernamental de la "coalición mayor". B. Las modificaciones más importantes en el derecho constitucional de 1987. III. RESUMEN Y PERSPECTIVA. 


\section{EL DESARROLLO DE LA CONSTITUCIÓN AUSTRÍACA EN LOS ÚLTIMOS DOS DECENIOS: REFORMA DE LAS CONSTITUCIONES}

POR

HEINZ SCHÄFFER

Catedrático de Derecho Público

Universidad de Viena

INTRODUCCIÓN

1. Esta ponencia sirve para la información sobre modificaciones importantes del derecho constitucional austríaco. Pero estas modificaciones se expondrán sobre todo con referencia a la reforma de las instituciones, un problema que domina también la discusión en la vida política.

Echando una mirada retrospectiva a los últimos dos decenios, el desarrollo se presenta ante todo como una serie de modificaciones puntuales del derecho constitucional (y de disposiciones en leyes ordinarias relacionadas con él). En Austria, las modificaciones constitucionales frecuentemente son producto de una "legislación ocasional», sin concepción a largo plazo, y a menudo resultan de las exigencias políticas del día. Sin embargo, se manifiesta que de estos cambios graduales resultaron nuevas estructuras y rasgos característicos del Estado y su constitución. Ciertos problemas de la vida política y de las instituciones siguen siendo discutidos hoy tanto como antes. Pero con el retorno al modelo gubernamental de la "coalición mayor" desde 1987, pasaron a primer plano parcialmente otros temas de política constitucional que en la era anterior. 
2. Notas en materia del modelo gubernamental y del proceso político, por lo tanto en materia de las instituciones políticas de por sí.

Se podría hacer la pregunta de por qué en los últimos años también se plantea la cuestión de la "gobernabilidad" en el sistema gubernamental de la Segunda República que durante mucho tiempo se consideraba un sistema estable. $Y$ es sólo una consecuencia de esta situación que ahora también en Austria se reflexiona sobre la reforma de las instituciones en alto grado.

Por eso, primeramente se expondrá cuáles eran las modificaciones en el sistema gubernamental y cuáles los cambios del sistema político que fueron realizados. La constitución prevé un clásico sistema gubernamental parlamentario con ciertos rasgos presidenciales. Esto significa que el gobierno federal, por cierto, se designa (formalmente) por el Presidente federal, pero -lo que tiene importancia decisiva en un sistema gubernamental parlamentario- depende de la confianza del Parlamento (jvoto de desconfianza!). Por lo tanto, el Presidente federal prácticamente no puede designar ningún gobierno que no tenga la mayoría en el Parlamento o por lo menos sea tolerado por una mayoría en el Parlamento. En esta situación constitucional se pueden imaginar varios modelos gubernamentales. Austria ya tenía todos estos modelos desde 1945:

De 1945 hasta 1947: gobierno de concentración (gobierno de todos los partidos: VP (partido popular) - SP (partido socialista) - KP (partido comunista) de 1947 a 1966: coalición mayor (VP-SP) de 1966 a 1970: gobierno monopartidista (VP) Ilamado "monocolor" de 1970 a 1971: gobierno minoritario (SP, con el apoyo implícito del pequeño FP (partido liberal) de 1971 a 1983: gobierno monopartidista (SP) de 1983 a 1986: coalición menor (SP-FP) desde 1987: otra vez coalición mayor (SP-VP)

Un gobierno en el sistema gubernamental austríaco puede ser estable solamente bajo ciertas condiciones básicas sobre todo en el paisaje partidista. Y precisamente, cuando son cumplidas las condiciones en cierto modo "matemáticas" para la formación de una mayoría: mayoría absoluta de un partido o si por los menos dos partidos disponen de una mayoría capaz de gobernar.

Para las coaliciones también es necesaria una homogeneidad politica suficiente para poder realizar un trabajo gubernamental común. No se cumplen siempre ambas condiciones.

La primera razón de ello consiste en que el derecho electoral ya no promueve mayorías. Más bien favorece partidos pequeños (no es que se 
trate de partidos mínimos) y de esta manera garantiza su representación en el Parlamento. (Los usufructuarios de esta regulación durante mucho tiempo eran la tercera fuerza politica en Austria, el FPÖ (partido liberal austríaco) y últimamente también el partido de los "verdes").

La segunda razón seguramente es más decisiva: los partidos tradicionales están sufriendo cierta congelación. Si ningún partido tiene la mayoría absoluta y si el clima de negociaciones entre los partidos (líderes) está sujeta a perturbaciones por causa de controversias, ataques personales y la toma de posturas de prestigio, entonces no pueden formarse ciertas constelaciones gubernamentales ni pueden solucionarse ciertos problemas reales importantes. Resulta un bloqueo general en el proceso político. La capacidad del sistema político de solucionar problemas se reduce. La disposición para arreglarse disminuye. (Tampoco la famosa "cooperación de los patronos y los obreros", que sigue siendo eficaz tanto como antes, no puede cambiar eso porque funciona solamente para ciertos tipos de problemasl.

Para ganar a partidarios fuera de su electorado fijo, los partidos clásicos se convirtieron en partidos colectivos y ellos, por su parte, en "partidos universales". (Eso vale especialmente para los grandes grupos políticos.) Sus programas son vagos y similares en muchos puntos; su política real a menudo es (independientemente de los programas) muy pragmática y oportunista.

Para ganar influencia, los partidos penetraron en muchos ámbitos sociales y efectúan un sistema de patronato muy corriente (distribución de puestos, adjudicación de viviendas etc.). Esta mala gestión económica y la tendencia táctica a lograr éxitos a corto plazo tienen por consecuencia que los partidos tradicionales no desarrollan ningunas perspectivas interesantes para los problemas futuros y parecen haber perdido en gran parte el sentido del cambio latente de los valores en la sociedad, aunque se sirven sistemáticamente del instrumento de la encuesta. Justamente del hecho de que ciertos nuevos problemas, situaciones de intereses y necesidades no fueron reconocidos (o no a su debido tiempo o no en la debida manera), resultó el nacimiento de los llamados "Nuevos Movimientos Sociales" y de muestras políticas de conducta fuera de los partidos. Pero las exigencias aún son más amplias y precisamente sobre todo en dirección hacia a) más "democracia directa" y b) más participación.

ad a) Democracia directa. En Austria existe no sólo la iniciativa popular como iniciativa legislativa sino también el referéndum popular (sobre las decisiones sobre leyes del consejo nacional). Ambas instituciones durante mucho tiempo sólo figuraban en los papeles; sólo empezaron a ser usadas desde 1964, pero no muy frecuentemente. 
De las hasta ahora diez iniciativas populares, sólo las tres primeras tuvieron éxito y motivaron leyes correspondientes: en 1964 tuvo lugar la iniciativa popular sobre la reforma de radiodifusión en la cual participaron 17,3 por 100 de las personas con derecho a votar (jinmediatamente 832.353 firmas!). En 1969 tuvo lugar la iniciativa popular sobre la introducción de la semana de 40 horas (participación: 17,7 por 100) y 1969 la iniciativa popular en contra del 13 año escolar (participąción: 6,78 por 100)

Quedaron sin éxito en 1975 la iniciativa popular para la protección de la vida humana (contra las nuevas disposiciones relativas al aborto provocado) la llamada "solución de plazo" (participación: 17,9 por 100), en 1980 la iniciativa popular en pro de la central nuclear en Zéentendorf (participación: 2,8 por 100) así como en 1982 la iniciativa popular en contra del centro de conferencias a pesar de 1.365 .000 votos (participación: 25,74 por 100). Una participación menor obtuvieron en el año 1985 la llamada «iniciativa popular Konrad Lorenz" (consagración de una ley fundamental a la protección del medio ambiente) con 353.908 votos (participación: 3,63 por 100 ) así como una iniciativa popular con el fin de la promulgación de una ley federal constitucional relativa a la celebración de un referéndum popular sobre la adquisición de cazas interceptores (iniciativa popular en contra de cazas interceptores -en pro de un referéndum popular)- obtuvo solamente 121.182 votos (participación: 2,24 por 100).

El único referéndum popular (regular) era aquél del año 1978 sobre el comienzo de la explotación de la central nuclear Zuentendorf. Según su resultado, fue decretada por el Parlamento una "ley antinuclear" que tiene vigencia hasta hoy.

Muchas veces, se ve en la democracia directa una posibilidad de vencer bloqueos políticos (situaciones de empate) y estructuras partidistas congeladas. El SPÖ (Partido Socialista Austríaco), como partido de cuadros, tradicionalmente toma una postura más reservada en cuanto a la democracia directa. Por otro lado existe también la siguiente idea de reforma, generalmente apoyada por la oposición respectiva: debe ser posible forzar un referéndum popular por medio de una iniciativa popular con apoyo masivo (más de $\mathbf{5 0 0 . 0 0 0}$ firmas) (= iniciativa de un referéndum). ¡Caso que una propuesta obtenga la mayoría en el referéndum, debería tener vigencia como una ley! Esta propuesta de reforma cambiaría la democracia representativa esencialmente, y aun la eliminaria parcialmente.

ad b) Participación: La discusión sobre la inclusión mayor de las personas afectadas en la elaboración de las decisiones administrativas ya dura algún tiempo. De esta discusión ya resultaron reformas en varios ámbitos (p.e. los amplios derechos de ser oído y derechos a manifestar su 
opinión en el ordenamiento territorial). Sin embargo, sería conveniente aumentar la información y los derechos de participación del ciudadano en muchos ámbitos. Eso debería estar previsto ya en la fase de planificación y no sólo cuando las decisiones ya fueron tomadas o prejuzgadas. Cuando el "ciudadano emancipado» no tiene ninguna oportunidad de enterarse a su debido tiempo de las planificaciones en el sector público y de manifestar sus intereses, pueden surgir conflictos enormes. La nueva conciencia democrática se manifestó (en diciembre de 1984) en la ocupación de la Hainburger Au (región a orillas del Danubio cerca de Hainburg) para evitar el comienzo de la construcción ilegal de una central eléctrica de gran potencia al borde del Danubio. Ciudadanos activos de cualquier convicción político-partidista, por medio de resistencia no violenta forzaron al gobierno a transigir y establecieron una nueva muestra de conducta política. Pero no será posible sin más introducir la participación en todos los campos, porque no se debe olvidar lo siguiente: la participación impide decisiones rápidas y admite en la elaboración de decisiones la consideración de intereses menos legítimos. Una participación suficiente en suma es más bien un problema de la reorganización graduada del derecho administrativo.

\section{LAS MÁS IMPORTANTES MODIFICACIONES CONSTITUCIONALES DESDE 1970. A MODO DE BOSQUEJO}

\section{A. Las modificaciones más importantes en la estructura constitu- cional}

1. El reglamento electoral para el consejo nacional de 1971. (Ley federal del 27-11-1970 sobre la elección del consejo nacional Bgbl (Boletín Oficial del Estado) $n .^{\circ}$ 1970/391)

En su triunfo electoral de 1970, Dr. Kreisíy, el presidente del SP, primeramente obtuvo solamente la mayoría relativa. Después de haberse asegurado de que el pequeño FPÖ (partido liberal austríaco) iba a tolerar su gabinete minoritario, tomó la iniciativa para modificar el reglamento electoral por lo cual al pequeño tercer partido - con el aumento del número de mandatos de 165 a 183 escaños-correspondía una representancia relativamente mayor. A base de las disposiciones electorales, el SPÖ obtuvo más tarde la mayoría absoluta tres veces. Cuando en 1983, se obtuvo solamente una mayoría relativa, eso significó para Kreisíy personalmente 
el final de su tiempo de gobierno. Pero era él, quien proyectó el sistema gubernamental de la coalición menor (SP/FP) con la predominancia del SP.

2. Desde el período antes de 1974, son de mencionar sólo algunas enmiendas constitucionales pequeñas, especialmente una que reguló los servicios auxiliares parlamentarios más en detalle (BVG [Ley federal constitucional] Bgbl 1973/391)

3. La enmienda de la ley constitucional federal (B-VGN) 1974 del 10-7-1974 Bgbl, 444

Era meta de esta enmienda de la constitución federal (B-VG) la realización parcial de un programa de reivindicaciones de los länder.

Con ello, se acabó esta larga tendencia que fue denominada "modificación total latente" (a cargo de los länder) de la Constitución. La enmienda de 1974 tendía a la ampliación de los derechos de los länder y la acentuación del "federalismo cooperativo».

La modificación más importante de 1974 la constituye el nuevo art. 15 a B-VG. Según esta disposición constitucional, la federación y los länder entre si pueden celebrar acuerdos sobre asuntos de su radio de acción respectivo (los llamados concordatos verticales y horizontales). Estos contratos también se llaman contratos de Estados-miembros o concordatos. Tales contratos se pueden contraer tanto sobre asuntos de la legislación como de la ejecución. Posibilitan una conducta armonizada (coordinada) de la federación y los länder incluso por encima de los límites de competencias fijados en la Constitución. Eso es de importancia especial en caso de las llamadas materias relacionadas con muchos ámbitos o materias complejas, las competencias de las cuales son distribuidas entre la federación y los länder (como p.e. ordenamiento territorial, protección del medio ambiente, reglamentación del mercado agrícola). Adicionalmente, fue introducido un control por el tribunal constitucional, si existen tales acuerdos y si fueron cumplidos. Otras exigencias de los länder, sin embargo, todavía no han sido cumplidas en 1974 (soberania financiera y revalorización del consejo federal). 


\section{Las cuatro enmiendas de la constitución federal del año 1975}

Se referían a diversos tipos de problemas:

a) La enmienda de la ley constitucional federal del 15-5-1975 BGBI 302. (la llamada "enmienda de la protección jurisdiccional»). Desde hace tiempo, se discutía la ampliación de la protección jurídica en el derecho público. La enmienda contiene las siguientes modificaciones:

aa) Solicitudes individuales en el procedimiento para el control de normas (según art. 139 y 140 B-VG).

A base del art. 139 apartado 1 y art. 140 apartado 1 B-VG, el tribunal constitucional ( $\mathrm{VfGH}$ ) resuelve sobre la ilegalidad de un reglàmento $\mathrm{o}$ además de la inconstitucionalidad de una ley también a solicitud de una persona que pretende haber sido afectada ( ella misma - no por medio de otras personas) directamente en sus derechos por la ilegalidad o inconstitucionalidad de la disposición legal, en tanto que la disposición legal correspondiente haya producido efecto para esta persona sin pronunciamiento de una decisión judicial o sin adopción de una decisión administrativa. Los siguientes tres presupuestos procesales deben ser cumplidos:

- El recurrente debe pretender haber sido afectado en un derecho subjetivo por la ilegalidad o inconstitucionalidad de la disposición legal. Para la legitimación de impugnación es de importancia decisiva la posibilidad de la lesión de un derecho subjetivo (el llamado "estado de ser afectado en la esfera jurídica").

- La lesión pretendida de los derechos subjetivos debe ser una lesión directa. "Directa" significa sobre todo que la disposición legal toma efecto para el recurrente sin pronunciamiento de una decisión judicial o sin decretación de una decisión administrativa. Eso se refiere a aquellos casos en los cuales una ley establece obligaciones del individuo ispo iure, sin que sea necesario primeramente aplicar en el concreto caso una ley o un reglamento ("medida-ley", "medida-reglamento»).

- La disposición legal debe haber tomado efecto, eso quiere decir que la disposición legal debe haber entrado en vigor (jviolación actual de la esfera del individuo!).

Adicionalmente, la jurisprudencia desarrolló otro presupuesto pro- 
cesal (por vía de la reducción sistemática-teleológica de la admisibilidad de la impugnación): el caso en que un rodeo jurídico no se puede exigir.

bb) Además, la enmienda trajo consigo la posibilidad de impugnar las llamadas "actuaciones materiales" en el Alto Tribunal administrativo $(V w G H)$. La regulación se refiere a los actos administrativos fuera de procesos, no formalizados, que afectan la posición jurídica del individuo. Hasta esta enmienda, el VfGH (tribunal constitucional), pero no el VéGH (tribunal contencioso-administrativo), consideraba y revisaba los llamados "actos oficiales fácticos" como decisiones. Ahora, al VéGH le fue concedida expresamente la competencia ( $y$ con ello fue obligado) a revisar el poder de mando y de coerción directo oficial (art. 131 a B-VG: recurso contra la medida). La competencia ya reclamada hasta ahora por el $\mathrm{VfGH}$ por vía de interpretación fue codificada espresamente en la B-VG (ley federal constitucional) (art. 144 B-VG).

cc) La ampliación del procedimiento de control de normas a solicitud judicial.

Mientras que la impugnación de leyes anteriormente era reservada solamente a las cortes más altas - al VéGH y a la Corte Suprema competente en lo civil y lo criminal-, por la enmienda constitucional de 1975 la posibilidad de impugnar leyes fue extendida a todos los tribunales competentes para la pronunciación de decisiones en $2^{\mathrm{a}}$ instancia. Con esta nueva regulación se llenó un hueco, porque la OGH (Corte Suprema) no es la última instancia en cada procedimiento judicial, y porque los otros tribunales tenían que aplicar leyes aun cuando se dudaba su constitucionalidad. Un presupuesto para este procedimiento de control de normas es el hecho de que el tribunal tiene que aplicar la norma respectiva (prejudicialidad = relevancia). Ya hasta ahora, el tribunal solicitante estaba obligado a solicitar al $\mathrm{VfGH}$ (tribunal constitucional) la revisión de la norma a aplicar, cuando ponía en duda la legalidad o la constitucionalidad de la disposición legal a aplicar.

Pero lo siguiente es algo nuevo: caso que la disposición legal a aplicar ya haya sido derogada, desde el año 1975 se puede solicitar al $\mathrm{VfGH}$ (tribunal constitucional) también la decisión que la disposición legal era ilegal o inconstitucional (art. $893 \mathrm{~B}-\mathrm{VG}$ ).

dd) La enmienda constitucional trajo consigo también una mejora de la situación de la minoría parlamentaria. 
Pero la enmienda constitucional no fortaleció solamente la posición del individuo ante el poder estatal, sino también la posición de la minoría parlamentaria concediéndole ciertos derechos fijados por leyes constitucionales. La enmienda del B-VG BGBI 1975/302 concede a la minoría parlamentaria, y precisamente a una tercera parte de los miembros del consejo nacional, el derecho a impugnar leyes en el VfGH (art. 140 parte 1 B-VG). Lo mismo se puede aplicar a los Parlamentos regionales si eso está previsto por la Constitución del land. Prácticamente se trata de un instrumento de la oposición cuya duda en la constitucionalidad de una ley fue ignorada por la mayoría simple.

ee) La no obligación a acatar instrucciones de las «autoridades colegiales con rasgos judiciales".

Según art. 133 inciso 4 B-VG, por leyes federales o leyes de los länder, pueden ser establecidas autoridades administrativas colegiales de la federación de los länder para las decisiones en última instancia en cuestiones administrativas de los entes territoriales. Estas llamadas «autoridades colegiales con rasgos judiciales" tienen una tradición jurídica cuyos orígenes se pueden encontrar en la época de la monarquía. Son proyectadas como autoridades especiales con capacidad de decisión en última instancia, similares a los tribunales contencioso-administrativos. Pero no son órganos supremos de la administración en el sentido del art. 69 aparte 1 B-VG, y por consecuencia no son órganos gubernamentales.

En la administración federal se pueden mencionar p.e. las siguientes autoridades: Comisión para la observación de la Ley sobre la radiodifusión, Comisión suprema en asuntos de patentes y marcas, la Alta comisión de servicio cívico, etc. Las decisiones de estas autoridades no están sujetas al control por el VéGH (tribunal contencioso-administrativo), si según la Ley de organización se encuentra entre los miembros por lo menos un juez, si fuera de ello, los demás miembros tampoco están obligados a acatar instrucciones desempeñando este cargo, si además las decisiones de la autoridad no están sujetas a la derogación o la modificación por vía administrativa y si -a pesar del cumplimiento de todas estas condiciones- el recurso al VéGH (tribunal contencioso-administrativo) no es expresamente declarado admisible por la ley. Por la enmienda constitucional de 1975, el art. 20 B-VG fue ampliado por un nuevo aparte 2. Según esta disposición, los miembros de las autoridades colegiales, que no sean jueces, ahora también ex lege no están obligados a acatar instrucciones desempeñando su cargo, si a tal autoridad colegial se le encarga la decisión en última, instancia a base de una ley federal ordinaria o una ley del land y si sus decisiones no están sujetas a la derogación o la modificación por vía administrativa, así como bajo la condición ulterior, que a estas autoridades 
pertenezca un juez. Según art. 133 inciso 4 B-VG, no es posible impugnar las decisiones de estas autoridades colegiales al VéGH (tribunal contencioso-administrativo), si la ley respectiva no declara admisible tal recurso expresamente.

Por consecuencia de esta modificación, la forma de organización especial de las "autoridades colegiales con rasgos judiciales" ahora son expresamente admitidas por el derecho constitucional como tipo especial en comparación con las disposiciones generales de organización de la administración constitucionales obligatorias (principio jerárquico).

b) La enmienda de la B-VG del 28-4-1975 BGBI. 316.

Esta enmienda introdujo una nueva distribución de competencias para el sistema de escuelas agrícolas y forestales.

c) La enmienda de la B-VG del 10-6-1975 BGBI. 368 (enmienda sobre la defensa nacional).

Con esta enmienda de la B-VG, el principio de la defensa nacional completa fue consagrado en la Constitución como objetivo legalmente fijado (fijación de un objetivo estatal). Junto con esta enmienda, el Parlamento adoptó una "doctrina de defensa" que fue dirigida al gobierno federal en forma de una resolución parlamentaria y expone los principios de la defensa nacional completa.

El sistema del servicio militar obligatorio fue formado por la Ley del ejército de $1978 \mathrm{BGBI} 150$ más en detalle, la prestación sustitutoria del servicio militar fue consagrada en una Ley de servicio cívico. La prestación sustitutoria del servicio militar fue construida como consecuencia de la libertad de conciencia y como derecho del ciudadano. Existe una comisión propia que resuelve sobre las razones morales cuya veracidad debe ser comprobada por el interesado.

En la nueva formulación de las tareas del ejército federal que no debe solamente defender las fronteras sino el territorio nacional fue considerada la imagen moderna de la guerra $y$ la idea estratégica de una defensa de todo el territorio.

d) La enmienda de la B-VG del 1-7-1975 BGBI 409.

Esta enmienda trajo consigo una pequeña reforma del Parlamento. 
El art. 12 aparte 4 B-VG sirve para la mejora de la situación de la minoria parlamentaria.

La disposición refundida da la posibilidad a una minoría cualificada de miembros del consejo nacional a encargar al tribunal de cuentas de llevar a cabo actos especiales del control de la gestión. Por lo tanto, esta regulación entrega a la oposición un instrumento de control efectivo. Según el reglamento interior, una tercera parte de los diputados puede exigir este control. Para evitar un abuso de este instrumento, una orden de control ulterior puede ser dada sólo después de que el tribunal de cuentas ha rendido un informe al Parlamento.

\section{La enmienda de la B-VG del 2-6-1977 BGBI 323}

Se refería sobre todo a modificaciones sobre la sustitución del Presidente federal y algunas otras disposiciones.

El Presidente federal sigue siendo representado tanto como antes por el Canciller federal. Tan pronto como el período de estar impedido dure más de 20 días, los tres Presidentes del Parlamento automáticamente serán nombrados un colegio para su representación. Ellos son destinados para la representación también cuando el Presidente no puede desempeñar su cargo permanentemente.

\section{La enmienda de la B-VG del 18-10-1977 BGBI 539}

La enmienda constitucional de 1977 trajo consigo una nueva regulación de la competencia de control del tribunal de cuentas, en cuanto a las "empresas dominadas" por el sector público. Con anterioridad a esta enmienda, la situación jurídica era la siguiente: el tribunal de cuentas tenía el derecho de control en empresas en las cuales la federación lo sea un land o un municipio) tenía participaciones financieras; pues en el caso extremo también cuando se trataba de una parte social insignificante de los entes territoriales mencionados.

Ciertas formas de la participación de sociedades, dominadas económicamente o de otra manera por el sector público, en el capital de otras sociedades no eran comprendidas suficientemente. Eso se refería sobre todo a las subparticipaciones de sociedades hijas y sociedades nietas del sector público así como a construcciones de consorcios y de holding. 
Por la enmienda, sin embargo, fue extendida la competencia de control del tribunal de cuentas a todas las empresas dominadas por el sector público hasta cierto grado de dominación. Cuando la federación, los länder y los municipios (los últimos en tanto que tengan por lo menos 20.000 habitantes) -o sea sólo o sea junto con otras entidades sujetas al control del tribunal de cuentas-, participen en el capital social nominal de tal empresa por un mínimo del 50 por 100, esta empresa debe estar sujeta al control del tribunal de cuentas, pero también las empresas de cada escalón (subparticipación) deben estar sujetas al control del tribunal de cuentas. Con esta formulación se logra que el tribunal de cuentas está facultado a controlar siempre cuando existe una influencia dominante del sector público sobre empresas.

\section{La enmienda de la B-VG del 22-2-1979 BGBI 92}

Esta enmienda sirvió para el desarrollo de la democracia. La edad para participar en las elecciones fue reducida otra vez: tienen el derecho a votar, los hombres y las mujeres que han cumplido en la fecha clave de las elecciones los 19 años de vida (art. 26 aparte 1 nuevo) y son elegibles aquellos que han cumplido en la fecha clave de las elecciones los 21 años de vida.

8. La enmienda de la B-VG del 13-3-1979 BGBI 134 - plasmó un primer paso hacia la llamada "reducción de privilegios" de la clase política

El fin de la refundición del art. 57 B-VG era la ampliación de la inmunidad profesional de los miembros de los cuerpos legislativos y al mismo tiempo la restricción de la inmunidad extraprofesional de los diputados a unos límites materialmente justificados conforme a la época. En ello, la inmunidad extraprofesional de los diputados tanto como antes es un obstáculo procesual a la persecución: eso significa: cuando se acaba la inmunidad, el caso puede ser investigado. Ahora, la autoridad puede perseguir actos punibles sin consentimiento del cuerpo representativo, cuando estos actos evidentemente no están relacionados con la actividad política del diputado. En otros casos, una persecución no debe ser realizada en tanto que el cuerpo representativo todavía no haya decidido; también se debe suspender la persecución, cuando el Parlamento lo exige. Este último tiene que resolver dentro de ocho semanas en todos los casos. Cuando el cuerpo representativo incurre en mora, la persecución puede ser efectuada. El tiempo durante el cual la inmunidad impide una persecución no debe ser 
incluido en el plazo de prescripción en el procedimiento penal judicial (art. 48 aparte 3 inciso 1 Código Penal).

\section{La enmienda de la B-VG del 1-7-1981 BGB/ 350}

Esta enmienda constitucional es un ejemplo muy típico de la técnica de modificaciones constitucionales puntuales (la llamada estrategia de mosaico). Se debe mencionar que esta enmienda fue preparada por una comisión de reforma constitucional establecida en la Cancillería federal (creada en 1975), pero que esta comisión mientras tanto expiró. Los contenidos principales de esta enmienda eran la introducción sin fecha fijada de la institución [defensor del pueblo] "abogacia popular" y una primera descarga del VfGH (tribunal constitucional).

El acuerdo sobre la promoción de la democracia directa consistía solamente en la reducción del número de los votos necesarios para una iniciativa popular de 200.000 personas con derecho a votar a 100.000 .

Una de las modificaciones más importantes es el control general de las irregularidades por la "abogacía popular». Desde el 1 de julio de 1977, en Austria existe una institución de protección jurídica en forma de la "abogacía popular" con una vaga semejanza a los Ombudsmänner (defensores del pueblo) escandinavos. La institución de la "abogacía popular" originalmente era un experimento. Pues, su existencia primeramente era limitada a un período de seis años (1977-1983). A base de las experiencias obtenidas entretanto, la "abogacía popular" fue convertida en una institución permanente del sistema de protección jurídica austríaco. La disposiciones básicas fueron insertadas en la B-VG (art. 148a-148j) como $7^{\mathrm{a}}$ pieza principal por la enmienda de la B-VG 1981 BGBI 350. La "abogacía popular" es un órgano colegial; consta de tres miembros eligidos por el consejo nacional que desempeñando su cargo son independientes e inamovibles. Eso debe garantizar que el control pueda ser efectuado sin presión e influencia de parte del Gobierno. Desde el punto de vista sistemático, en el caso de la actividad de la "abogacía popular" de ejercer el control de irregularidades no se trata de administración, más bien de un control de la administración por un órgano auxiliar del poder legislativo. El poder de control es muy amplio; ya que el control -como lo prevé la ley-se refiere a todas "las irregularidades en el campo de la administración federal». Ahí son incluidos tanto el campo de la administración soberana como también el campo de la administración de la economía privada de la federación (pero según la doctrina dominante está incluida solamente la administración de la economía privada de la federación misma, pero no la gestión de 
las entidades desincorporadas, como por ejemplo empresas y sociedades dominadas). Una ley constitucional de un land puede declarar la «abogacía popular» competente también para el campo de la administración regional, lo que sucedió hasta ahora en siete de nueve länder (hasta ahora no en Tirol y Vorarlberg).

En cuanto al modo de la lesión jurídica, el control por la «abogacía popular" abarca un amplio espectro empezando de las lesiones de derechos públicos subjetivos del individuo pasando por la lesiones jurídicas objetivas hasta cualquier "comportamiento incorrecto de los órganos administrativos" frente al individuo. Como "irregularidad" se considera un amplio concepto que contiene un comportamiento incorrecto de la administración que no es en primer lugar un comportamiento incorrecto jurídico; más bien, se trata del control de un comportamiento que está en contradicción con los buenos modales, la equidad, la formalidad y el principio de una administración oportuna. Por eso, el concepto de la irregularidad no se puede definir, pero sí que se puede describir tipológicamente. Un individuo tiene la legitimación de quejarse, si está afectado por una uirregularidad pretendida" de la administración y si ya no tiene ninguna posibilidad de interponer un recurso contra ello (jincluso, cuando no observó el plazo del recurso!). La "abogacia popular" está obligada a investigar cada queja y comunicar al recurrente los resultados de la investigación así como las medidas acaso adoptadas. La "abogacía popular» puede investigar irregularidades presumibles dentro de la administración aun de por sí (es decir, de oficio). Todos los órganos estatales deben apoyar a la uabogacía popular" en su actividad de investigación como es debido; así por ejemplo, tienen que entregar los autos o dar cualquier otro informe. La "abogacía popular" misma no dispone de ningún medio sancionador; solamente está facultada para dar «recomendaciones» jurídicamente no obligatorias: una especie de soft laé constitucional. Considerando el efecto de tal recomendación, ciertamente no se debe olvidar la buena reputación de la institución, que hace casi imposible para la autoridad administrativa no corresponder a tales recomendaciones. Finalmente, la "abogacía popular» tiene que rendir un informe sobre su actividad al Parlamento cada año. La segunda demanda principal de la enmienda de la B-VG BGBI 1981/350, sin embargo, era la regulación de la llamada descarga del VfGH. Algo totalmente nuevo para el orden jurídico austríaco era una disposición en relación con el recurso de inconstitucionalidad. Al $\mathrm{VfGH}$ le fue conferido el derecho a rechazar un recurso (a limine), "cuando no tiene suficiente probabilidad de éxito". (No se debe rechazar la tramitación, cuando se trata de un caso que según art. 133 B-VG está excluido de la competencia del VéGH.)

Pero esta posibilidad mencionada del rechazo no cortó a aquellos 
que buscan protección jurídica el camino a un tribunal supremo. El recurrente puede solicitar que el VfGH transfiera el recurso al VéGH en caso de rechazarlo, para que éste decida si el recurrente ha sido lesionado por el acto impugnado en otro derecho.

\section{La enmienda de la B-VG del 2-3-1983 BGBI 175}

Cuya meta era la creación de una competencia de la federación en el ámbito de la protección del medio ambiente. Esta enmienda es el resultado de largas negociaciones entre la federación y los länder, que se expresó en la creación de una nueva competencia federal para la protección contra inmisiones a cambio de algunas modificaciones deseadas por los länder.

A base de la refundición del art. 10 aparte 1 inciso 12 B-VG, la competencia a adoptar medidas de protección contra peligros para el medio ambiente que resultan del exceso de valores límites de inmisiones, ahora corresponde a la federación. Bajo la condición de una carga peligrosa, la federación no es competente sólo para el hombre y su entorno ecológico, sino también para el medio ambiente en general, es decir, para la legislación y la ejecución de todas las medidas necesarias y adecuadas para la protección contra estos peligros. A base de esta nueva competencia, en el futuro será posible hacer frente a concretos peligros del medio ambiente por medio de medidas adecuadas; pero en el campo de la política "profiláctican del medio ambiente sigue realizándose la política del medio ambiente que se realizaba hasta ahora (a base de competencias especiales distribuidas). Según art. II de la enmienda de la B-VG, una ley federal que se apoya en la nueva competencia federal puede ser decretada solamente bajo la condición de que existe y ha entrado en vigor un convenio correspondiente entre la federación y los länder según art. 15 B-VG sobre los valores limites de las inmisiones que se deben regular por leyes federales. (Las disposiciones del art. II no tienen vigencia para las medidas en los ámbitos de la protección especial del medio ambiente que correspondían a la federación hasta ahora.)

\section{La enmienda de la B-VG del 26-6-1984 BGB/ 296}

La enmienda constitucional de 1984 sirvió para una nueva descongestión de ambos tribunales de derecho público (VfGH, VéGH). Por el acortamiento de la duración del proceso, se debe garantizar además la posibili- 
dad para una rápida elaboración de las decisiones que constituye un elemento esencial de la protección jurídica. La base para esto era un informe del Canciller federal relativo a las posibilidades de una nueva descarga de los tribunales de derecho público. (Había el peligro, que las instancias de Estrasburgo del Convenio Europeo de los Derechos Humanos pusieran dificultades a Austria en algunos casos por causa de una duración del proceso extralarga.)

Ahora ya no es posible presentar quejas por demora de la decisión (art. 132 B-VG) al VéGH en asuntos penales administrativos (a excepción de los asuntos de acusación privada y asuntos penales financieros). Adicionalmente, una enmienda de la Ley penal administrativa (BGBI 1984/299, art. 51 aparte 5) determinó que la decisión recurrida se considera como abolida ex lege y que se debe sobreseer el procedimiento cuando la autoridad de apelación no decide dentro de un año.

Para simplificar el proceso aún más, ahora decide una sala de solamente tres jueces (ya no una sala de cinco jueces) sobre las sanciones administrativas en el VéGH (art. 11 aparte 1 frase 1 VéGG Ley del tribunal contencioso-administrativo). La decisión sobre el reconocimiento del efecto suspensivo aún se encarga a un juez solo (art. 14 VéGG).

Además, para el tribunal constitucional fueron ampliadas las razones por las cuales éste tiene el derecho de rechazar la tramitación de un recurso. La refundición del art. 144 aparte 2 B-VG corresponde a las propuestas presentadas por el $\mathrm{VfGH}$ mismo. Ahora el rechazo de la tramitación de un recurso aún es admisible, "cuando de la decisión no se puede esperar la aclaración de una cuestión constitucional». La protección jurídica del individuo sigue siendo garantizada en cierto grado, porque un recurso no debe ser rechazado en el caso de que el asunto fuese excluido de la competencia del VéGH. Por consiguiente, de todas maneras se puede acudir a uno de los dos tribunales.

12. La enmienda de la B-VG del 10-12-1984 BGBI 490 realizó otro cumplimiento parcial del programa de demandas reividicativas de los Länder

A finales de 1984, de nuevo se realizaron pequeños mejoramientos del federalismo y la consagración de una fiajación más amplia de las metas estatales. a) Es de importancia teórica y práctica que en el futuro las modificaciones de las competencias a cargo de los länder sólo se realizarán con el consentimiento cualificado del consejo federal (se requiere un 
quorum de $1 / 2$ de los miembros y una mayoria de $2 / 3$ de los votos) (art. 44 parte 2 B-VG). b) La enmienda creó además una legislación de emergencia también a nivel regional. c) Para la protección del medio ambiente fue creada una ley federal constitucional propia (BVG BGBI 1984/491) que, por cierto, destaca con un "credo" la importancia política de la protección del medio ambiente, pero no tiene ningún valor normativo: no tiene ningunas consecuencias jurídicas directas. Solamente expresa que el Estado se da cuenta del problema y quiere dedicarse más a esta cuestión.

\section{La enmienda de la B-VG 1986 BGBI 212}

Esta enmienda de la Constitución federal finalmente trajo consigo la reorganización del derecho presupuestario discutida hace ya dos decenios. Un mandato constitucional (art. 13 aparte 2 B-VG) obliga a los entes territoriales (esos son la federación, los länder y los municipios) a tender al equilibrio económico general en su gestión presupuestaria. Eso es una alusión a las metas reconocidas por las ciencias económicas y financieras modernas de una política de estabilidad (el llamado "polígono mágicon), como son consagradas p.e. en el derecho presupuestario de Suiza y en la ley de estabilidad de la República Federal de Alemania. Los puntos centrales de la enmienda eran el aumento de la flexibilidad en la ejecución presupuestaria, la amplia reorganización del estado provisional presupuestario y una fundación constitucional de la política de perecuación coyuntural (del llamado stop and go). Además, fue instalado un control parlamentario que acompaña la ejecución presupuestaria. Se refiere, por un lado, a las medidas presupuestarias de actuación conyuntural; por otro, a las extralimitaciones presupuestarias durante el año fiscal. La cooperación entre el ministro de Hacienda y el Parlamento consiste, de una parte, en la obligación a rendir informes (por trimestre) y, de otra, en el verdadero acuerdo.

Estos principios constitucionales fueron concretados más en detalle en una ley federal presupuestaria propia (BGBI 1986/213) que también introdujo una planificación financiera a medio plazo, que sirve, sin embargo, solamente para la orientación (y por consecuencia, no es obligatoria).

\section{B. Las tendencias principales del desarrollo de la Constitución hasta 1986 - reforma de las instituciones}

En el desarrollo de la Constitución de esta época se pueden percibir algunas tendencias principales. 


\section{La ampliación del federalismo}

Lo que más salta a la vista, es una animación del federalismo en Austria. Los länder se dan cuenta de su estatalidad en grado más alto. Reformaron sus constituciones regionales y presentaron a la federación un programa de exigencias con el fin de la ampliación de sus derechos regionales. Este programa de exigencias fue realizado parcialmente en varias etapas. En ello, la federación ganó a cambio del cumplimiento de algunas exigencias de los länder una competencia central en la protección del medio ambiente, pero no confirió ninguna competencia de verdadera importancia a los länder. En total, se puede decir que Austria - a pesar de algunas características cooperativas-sigue siendo un Estado federal más bien unitario.

2. Ampliación y reestructuración de las instituciones de protección juridica y de control.

Un segundo punto esencial del desarrollo se refería a la ampliación y la reestructuración de las instituciones de protección jurídica y de control. Un refinamiento mayor y un mayor perfeccionamiento fueron logrados por desarrollos ulteriores del sistema de la protección jurídica que son conformes con el sistema.

Así por ejemplo, el VéGH tiene que controlar además de las decisiones también actuaciones de la administración fuera de procesos (no formales). $Y$ el control de las normas (de leyes y reglamentos) por el tribunal constitucional fue ampliado a tratados entre Estados y repronunciaciones.

Mientras que los instrumentos tradicionales de la protección jurídica se perfeccionan, se manifestó un nuevo deseo de los ciudadanos de protección contra acciones estatales no formalizadas y el deseo de una administración adecuada para los ciudadanos y orientada hacia el servicio. Para este deseo fue -mencionado ya- «implantado" en nuestra Constitución un nuevo órgano constitucional según ejemplos extranjeros: la llamada "abogacía popular" como tipo de Ombudsmann colegial o sea mediador a la administración. Es una respuesta por ahora todavía imperfecta al deseo de un control que sea informal, pero eficiente, en los ámbitos no formalizados de la actividad estatal. 
3. La modificación de la Constitución según contenido, estilo y técnica de creación del derecho

a) La modificación del derecho constitucional se realiza en muchos pequeños pasos y no sistemáticos según las necesidades de la política cotidiana y sin gran concepción ("estrategia de mosaico»). Eso perjudica la claridad y también la consistencia interna del derecho constitucional. Hay críticos que ya hablan de un "carácter ruínoso de la Constitución federaln. Los pocos intentos irresolutos de una reforma constitucional (p.e. trabajos preparatorios por una comisión de la reforma constitucional) fracasaron por falta de interés efectivo de parte de los partidos políticos en las cuestiones fundamentales de la política constitucional.

b) Ultimamente, se introducen mandatos constitucionales poco precisos (una especie de soft laé), lo que está en contradicción con la tradición del derecho constitucional austríaco.

Tales textos contienen solamente un credo de ciertos valores fundamentales y pueden ser considerados como mandatos constitucionales al legislador ordinario relativamente imprecisos, pero no como derecho constitucional directamente aplicable (ni siquiera órdenes constitucionales). Como ejemplos se pueden mencionar el credo de la defensa nacional universal (1975) y de la protección del medio ambiente universal (1985).

c) Finalmente, hay una tendencia a integrar reglas en la constitución sobre asuntos que anteriormente se regularon por el libre juego de las fuerzas políticas o por la convención.

En la Ley sobre los partidos de 1975 BGBI 404, los partidos políticos, que durante decenios actuaban en un vacio jurídico, fueron reconocidos expresamente en la Constitución como partes esenciales de la democracia. Por lo demás, la formulación de la ley excluye un sistema propio de admisión o un sistema de prohibición.

En pequeños pasos, los derechos de control parlamentarios fueron ampliados de manera que en dos puntos prácticamente se puede hablar de un reconocimiento constitucional de la oposición, aunque no en forma jurídica: desde entonces, una tercera parte de los miembros de un cuerpo parlamentario a nivel federal y regional (consejo nacional, consejo federal, parlamento regional) puede solicitar la revisión de una ley al VfGH. Cada vez una tercera parte de los miembros del consejo nacional puede dar al tribunal de cuentas una orden obligatoria de revisión (frente a la administración dirigida por el gobierno). 


\section{Ideas y tendencias de reforma de la época 1970 a 1986}

Aparte de diversos deseos de reforma constitucional que también han sido mencionados en la literatura científica (depuración jurídica, por lo menos del derecho constitucional, ampliación del federalismo, democratización, participación, despolitización del tribunal constitucional, reforma de los derechos fundamentales) se discutían dos grandes ciclos temáticos.

Concretas propuestas de reforma, por un lado, se referian esencialmente a deseos de la oposición inmanentes al sistema. Por otro lado, durante algún tiempo se reflexionaba sobre la consagración constitucional del modelo del "gobierno de concentración".

\section{EL ÚLTIMO PERÍODO DE 1986-1989 (HASTA HOY)}

\section{A. Retorno al modelo gubernamental de la "coalición mayor"}

Una situación por varias razones crítica acarreó el final de la "coalición menor", pero, sin embargo, no un "cambio" (conservativo). El cambio súbito del líder del FPÖ tuvo por consecuencia el fracaso de la "coalición menor", y ambos grandes partidos consideraban el FPÖ como no capaz de pactar. Bajo estas circunstancias, después de las elecciones de 1986 como única posibilidad política a base de la distribución de los mandatos en el Parlamento se ofreció una cooperación de los grandes partidos, porque ninguno de éstos disponía de un numero suficiente de mandatos por si sólo. Esta cooperación también era necesaria desde el punto de vista material para la solución de graves problemas, como especialmente el saneamiento del endeudamiento del Estado y de la industria nacionalizada casi quebrada. Este gobierno paso a paso empezó a solucionar problemas a nivel constitucional, que requerían una solución ya hace mucho tiempo.

- ¿Cómo funciona este mecanismo gubernamental de la coalición mayor políticamente?

Ya que según la práctica constitucional austriaca, las decisiones del gobierno federal deben ser adoptadas por unanimidad, la coordinación previa tiene una importancia esencial. Con pleno conocimiento, en el acuerdo de trabajo no fue creada una "comisión de coalición" para evitar 
desarrollos paralelos con la coalición mayor pasada (antes de 1966), en tanto que sus prácticas hoy día se consideren como errores. No se debía causar la impresión que los partidos crearían un órgano extraconstitucional con la capacidad de última decisión (órgano supremo). Tampoco debía ser coartada la cooperación en la coalición por reglamentos. Sin embargo, en la Cancillería federal fueron designados dos llamados «ministros de la Cancillería", uno del SPÖ y uno del ÖVP; por cierto, se dedican también a cuestiones materiales, pero desde un principio, como su función politica se consideraba el fomento de la cooperación. En la práctica se formó además una comisión coordinadora de seis miembros que se extiende en casos importantes a la participación del Canciller federal (SPÖ) y del Vicecanciller (ÖVP). Este gremio discute el orden del día del Consejo de Ministros, pero también otras cuestiones políticas que requieren un consentimiento, como p.e. iniciativas parlamentarias y cuestiones del procedimiento parlamentario. El consentimiento de ambos lideres de los partidos (Canciller federal y Vicecanciller) en todos los asuntos tiene una importancia esencial. Todos los lunes, tienen lugar conferencias preliminares de la fracción gubernamental, y los martes tiene lugar una conferencia preliminar del Consejo de Ministros antes de la sesión del gobierno federal. En esta sesión se lleva a cabo el debate material; los puntos sobre los cuales no se puede llegar a un acuerdo no se discuten más. La sesión oficial del Consejo de Ministros es una pura formalidad, dura muy poco tiempo y solamente sirve para la protocolación de los acuerdos adoptados en la conferencia preliminar.

Esta coalición mayor no cumplió diversas tareas de su programa de ambicioso trabajo, como, p.e., reforma de la democracia, reforma de las pensiones y mejoramientos esenciales en la salud pública, en el mundo laboral $y$ en el sector de la protección del medio ambiente.

\section{B. Las modificaciones más importantes en el derecho constitucional de 1987}

1. La enmienda de la B-VG BGBI 1987/286. Se refería al deber genérico de información de la administración.

Desde esta enmienda, todos los órganos ejecutivos estatales ( $\mathrm{V}$ también los órganos ejecutivos de otra corporación de derecho público) tienen que dar informe a petición sobre los asuntos de su radio de acción, en tanto que no exista una obligación legal de guardar el secreto. Este deber de información general debe poner la ejecución más al alcance de los ciudadanos. 
2. Por estas disposiciones constitucionales (parcialmente en la Ley de financiación de ÖIAG BGBI 1987/298, parcialmente en la segunda Ley de nacionalización BGBI 1987/321) fueron establecidas las condiciones de una privatización (parcial) de empresas nacionalizadas. En la economía de electricidad nacionalizada, 51 por 100 de las acciones deben quedarse en posesión del sector público también en adelante (que por eso sigue teniendo un llamado "núcleo duro»). Disposiciones constitucionales que impidieron la enajenación de participaciones en otras empresas estatales (industria, bancos) fueron derogados sin sustitución alguna. La cuestión de si la privatización parcial necesaria para la obtención de capital nuevo puede exceder el 51 por 100 en este sector, es una cuestión política todavía no decidida.

\section{La enmienda de la B-VG BGBI $1987 / 640$}

Con esta enmienda fue cumplida una exigencia de muchos años de los länder.

Les fue conferida la competencia del fomento de la construcción de viviendas $y$ del saneamiento de viviendas, así como la competencia de los asuntos civiles relacionados con esto.

\section{La enmienda de la B-VG BGB/ 1988/341}

Con esta enmienda, la posición del consejo federal como segundo cuerpo de nuevo fue revalorizada un poco. En primer lugar, el consejo federal como órgano que participa en la legislación de la federación también obtuvo el derecho a solicitar -con un mímino de una tercera parte de sus miembros - la revisión de leyes al tribunal constitucional. Además, por esta modificación constitucional fue consagrado el llamado sistema de milicia en la Constitución federal.

5. Una de las más recientes, la enmienda de la B-VG BGBI 1988/685

La gran enmienda constitucional tenía varios puntos centrales: federalismo, reforma de la democracia, control.

a) Desde el punto de vista federalista, la enmienda trajo consigo 
un cumplimiento parcial ulterior del programa de reivindicaciones de los länder. Es de importancia práctica y de interés para la teoría del Estado, que a los länder fue conferida una competencia jurídico internacional de concluir contratos con Estados vecinos. Los länder pueden concluir contratos internacionales con Estados vecinos o Estados-miembros de ellos en los asuntos de su radio de acción. Antes de entrar en negociaciones, se debe pedir la aprobación del gobierno federal; pero esta aprobación se presume, si no se rehusa expresamente dentro de ocho semanas.

En cambio, la federación obtuvo varias competencias en el sector de la protección del medio ambiente; en primer lugar de la purificación de aire (excepto instalaciones de calefacción), de la economía de desechos (y precisamente sin límites en cuanto a desechos peligrosos, por lo demás se trata de una "competencia en caso de necesidad" en tanto que exista una necesidad de regulaciones unificadas) y, además, una "competencia en caso de necesidad" de la fijación de valores límites de emisiones unificados para sustancias contaminantes del aire laquí también en tanto que exista una necesidad de regulaciones unificadas).

b) Otro punto central se refería a cuestiones de la reforma de la democracia. Una iniciativa popular Lapoyada por un mínimo de 100.000 personas con derecho a votar o por $1 / 6$ de las personas con derecho a votar de tres (änder) debe, por cierto, referirse a un asunto de la legislación federal, pero en el futuro ya no tiene que presentar un proyecto de ley formulado en detalle. Además, el instrumento de la consulta popular fue introducido también a nivel federal, pero sólo para "cuestiones de importancia fundamental y relativas a toda Austria" que corresponden a la competencia de regulación del legislador federal. También es interesante el hecho de que la federación de ciudades y la federación de municipios como asociaciones de intereses de los municipios austriacos (y con ello directamente de su población) fueron consagradas en la Constitución.

c) Un tercer punto central de la gran enmienda constitucional de 1988 se referia a las diversas instituciones de control. La competencia de control del tribunal de cuentas fue ampliada y precisada. A las tareas que tenía hasta ahora se agregó el control periódico de la situación de los ingresos de los empleados de empresas públicas y la información del Parlamento sobre eso.

Mientras que la competencia de control de la "abogacía popular» hasta ahora se refería solamente a órganos administrativos estatales, ahora le fue conferida también una competencia de recomendación en cuanto a los órganos autónomos y autoridades administrativas no obligados a acatar instrucciones. Para una nueva orientación fundamental del control 
administrativo judicial que entró en vigor a partir de 1991, fueron previstas "salas administrativas independientes en los länden. El motivo directo para la creación de estas instituciones de concepción nueva para Austria era la jurisprudencia del Tribunal Europeo de Derechos Humanos de Estrasburgo que precisó en varias decisiones ciertas exigencias de independencia de aquellos tribunales que tienen que resolver sobre asuntos civiles y penales (en el sentido del art. 6 MRK - Convenio de Derechos Humanos). Para cumplir estas exigencias del Convenio sobre todo en el campo del derecho penal administrativo, pero también de aquellos asuntos administrativos que según la jurisprudencia de las instancias de Estrasburgo pertenecen al amplio concepto del derecho civil, el legislador constitucional ahora previó órganos propios de control administrativo similares a los tribunales. En principio, se trata de una especie de seudojurisdicción administrativa, ya que uno puede recurrir a estas salas, solamente después de haber agotado todas las instancias administrativas; en principio, se trata de instancias del control jurídico. Ya que son órganos administrativos, sus decisiones además están sujetas al control posterior por el Alto Tribunal Administrativo. Así fue constituida casi una jurisdicción administrativa con varias instancias. (La formación detallada corresponde a la legislación regional.)

6. La reforma del reglamento parlamentario por la enmienda (del GOGNR - Ley sobre el reglamento del consejo nacional)- BGBI $1988 / 720$

La gran reforma del reglamento interior del consejo nacional también forma parte del desarrollo ulterior del derecho constitucional en el sentido material. En primer lugar, se referia a mejoramientos técnicos y una animación del parlamentarismo. Ciertos asuntos discutidos se pueden llevar al pleno en un proceso acortado (es decir, sin debate en la comisión). Conforme al sistema del Estado multipartidista, los clubs de los partidos parlamentarios delegan a los miembros de la comisión (ya no tiene lugar una elección en el sentido más estricto). La introducción de una "publicidad parcial" en las comisiones investigadoras se considera muy importante; es decir, que representantes de los medios (periodistas) pueden estar presentes por acuerdo de la comisión investigadora, pero no se admite hacer grabaciones de televisión y de radiodifusión ni fotografías. Para animar los debates, el tiempo asignado a cada orador fue limitado por mutuo acuerdo. Algo nuevo son además la llamada "hora actual" (según el ejemplo del Bundestag (Parlamento alemán) así como uencuestas parlamentarias" (corresponde a los Hearings) y las llamadas "comisiones de encuesta" (comisiones periciales para la elaboración de diversos temas). 
En lo futuro se aplicará un nuevo reglamento detallado a las peticiones e "iniciativas parlamentarias de ciudadanos" (peticiones de un mínimo de 500 ciudadanos al Parlamento en el cual un representante de la iniciativa puede participar en los debates de la comisión).

\section{RESUMEN Y PERSPECTIVA}

Cuestiones de la política constitucional no solucionadas.

Las cuestiones de la política constitucional no solucionadas se refieren sobre todo a las crecientes interdependencias internacionales de las decisiones estatales, el desarrollo ulterior oportuno del federalismo y en primer lugar los problemas de la democracia.

\section{El Estado en la red de las relaciones internacionales}

Teniendo en cuenta que en Austria actualmente por lo menos una cuarta parte de todos los actos legislativos tienen un origen internacional y muchos actos intraestatales quedan influenciados esencialmente por compromisos internacionales contraídos, se plantea la cuestión de una reorganización de los procedimientos y de la legitimación del poder externo. Este problema se agravaría aún más en el caso del ingreso de Austria en la CE e incluso en caso de una mera asociación. Se debería establecer una base especial constitucional para el derecho de integración. Además, se deberian encontrar soluciones para la pérdida de importancia e influencia de los länder dentro de la integración europea (de manera semejante como en la República Federal de Alemania).

\section{Federalismo}

Desde hace muchos años, se exige de la doctrina y de los länder que se encuentre una nueva distribución de competencias de todas las tareas estatales entre la federación y los länder una distribución orientada por principios claros que no admite alusiones y concurrencias. Eso se refiere sobre todo a una clara distribución de las responsabilidades en la administración no soberana («administración de economía privada»), es decir, en el campo de las prestaciones, fomentos y empresas públicas. 
Además, tal nueva distribución de las responsabilidades debería ser organizada análogamente en la Constitución financiera. Eso significa: los länder- $y$ a nivel inferior: los municipios- deberian obtener suficientes derechos impositivos o participaciones debidamente garantizadas en los ingresos del nivel territorial superior respectivo.

\section{Problemas de la democracia}

Las problemas más importantes, por supuesto, consisten en ciertos síntomas de cansancio y problemas estructurales de la democracia representativa.

a) Aunque en el acuerdo de trabajo de 1987 de la coalición mayor fue prometida una personalización del derecho electoral con el fin de la consolidación de la democracia, hasta ahora no fue realizada tal personalización. Originalmente, fue planeado el abandono parcial del orden electoral de listas (donde se eligen listas) que a través del establecimiento de las listas de candidatos ha aumentado la influencia de los secretariados de los partidos. Debería ser posible eligir en, aproximadamente, 100 distritos electorales -en vez de los nueve distritos electorales que existen actualmente- a un candidato de partido por medio de un voto en pro de un partido y a otro candidato (que bajo ciertas condiciones ni siquiera aparece en esta lista) por medio de un voto de preferencia. Evidentemente, el miedo de cambios personales de parte.de los funcionarios de los partidos evitó esta reforma.

b) Ya desde algún tiempo se está discutiendo a nivel federal una prolongación del período legislativo del Consejo nacional de cuatro a cinco años, aunque actualmente ya no se habla mucho sobre eso. (En varios länder, los parlamentos regionales tienen mandatos que duran cinco años, pero a eso hay que añadir que en la mayoría de los länder existe una obligación constitucional de cooperar en forma de un gobierno de concentración.) Una crítica justificada del sistema gubernamental parlamentario se refiere al hecho de que los políticos muy frecuentemente piensan solamente en la corta dimensión temporal de un periodo legislativo y que por eso decisiones en aquellos campos que necesitan una orientación a largo plazo (como sistemas de la seguridad social, política de energía y de la protección del medio ambiente, etc.) no pueden ser adoptadas a su debido tiempo o no con la bastante responsabilidad o no pueden ser adoptadas de ninguna manera.

«Falta la previsión a largo plazo». En realidad, al principio de un pe- 
ríodo legislativo todos se dedican a la busca de un gobierno capaz de trabajar $y$ al menos un año antes de terminar este período a la creación de un perfil propio para la próxima lucha electoral. El tiempo para el trabajo constructivo es corto. Según la opinión de aquellos que apoyan la prolongación del período legislativo, tal prolongación permitiría al Parlamento trabajar de manera más tranquila y más práctica. Pero a mi parecer, se olvida que un período legislativo normalmente se puede agotar sólo cuando existe un gobierno capaz de actuar. Cuando el gobierno es un gobierno de coalición y una vez agotada su mutua conformidad, según la costumbre de la democracia parlamentaria, de todos modos no hay más remedio que celebrar nuevas elecciones.

c) Un problema prinicipal constituye - también en Austria- un descontento con los partidos políticos muy generalizado. La investigación social empírica sabe eso ya desde algún tiempo y recientemente este descontento se expresa aun en decisiones electorales masivas. (¡Así p.e. en la última elección del consejo nacional un 20 por 100 y en la última elección del Parlamento regional de Viena hasta un 40 por 100 de los electores no participaron en las elecciones!). El enojo se refiere sobre todo a acumulaciones de cargos, a la toma de influencia ilegal que sólo ahora se están descubriendo poco a poco y a privilegios considerados injustos de un grupo de funcionarios de los partidos. Al menos hay tendencias a una desconcentración y al desarrollo de una nueva moral política, aunque los partidos y sus funcionarios evidentemente siguen temiendo la transparencia y la publicidad absoluta. No hay que perder de vista que los partidos políticos en una democracia parlamentaria constituyen un elemento irrenunciable del sistema político.

Amplios grupos de la población desean, sin embargo, una reducción de influencia de los partidos políticos en la vida pública, porque ya ganaron influencia en demasiados ámbitos de vida (por ejemplo en la distribución de puestos, adjudicación de viviendas etc.). Pero la reducción de esta influencia no parece realizable. Bajo el título de la objetivación se está elaborando una distribución de las esferas de influencia de modo que en muchos casos las posibilidades profesionales de las personas sin partido político son reducidas. Pero un acuerdo de los partidos sobre incompatibilidades y disposiciones de declaración económicas y políticas más extensas ya sería una gran ventaja, así como el establecimiento de ciertas reglas fundamentales para los partidos en cuanto a su orden interior en la Ley sobre los partidos. Claro está que es muy difícil conseguir eso porque en cierto modo se trata de una restricción y un orden de buena conducta del cartel de poder.

d) Otro problema estructural importante de la democracia moder- 
na constituye el status insuficientemente definido de los medios de comunicación de masas. Con mucha razón se denominan muy frecuentemente el "cuarto poder». Los medios de comunicación de masas, sobre todo la televisión, profundamente cambiaron las posibilidades de acción de los actores e instituciones del sistema político. En la medida en que los medios establecen una publicidad $y$ descubren irregularidades, ejercen una función importante de crítica. Pero normalmente no pueden realizar ninguna política constructiva propia.

\section{Mecanismos de una coalición y condiciones para una política a largo plazo}

La reciente historia austríaca ofrece un material ilustrativo muy bueno para los rasgos característicos de una "coalición menor" y una "coalición mayor".

En una coalición menor, el pequeño coalicionista de todos modos es necesario para obtener la mayoría. Si tiene una influencia más amplia y decisiva en la línea política del gobierno, finalmente depende de los personajes. Un pequeño coalicionista prudente puede, en ciertas circunstancias, conseguir del coalicionista mayor concesiones relativamente amplias. Pero en Austria, el período de 1983-1986 no puede ser comparado con la "época social-liberal» de la República Federal de Alemania.

El caso es absolutamente otro en una "coalición mayor" con coalicionistas aproximadamente equilibrados, como la tenemos actualmente. La formación y la existencia más larga de este modelo gubernamental dependen de un consentimiento fundamental sobre, al menos, algunas cuestiones importantes y de la disposición para cooperar durante algún tiempo. El consentimiento no debe referirse solamente a algunos puntos y a corto plazo. Según las experiencias anteriores de Austria, la obligación de ponerse de acuerdo en una coalición no puede ser absoluta. En materias sobre las cuales no se han tomado acuerdos, también es posible una iniciativa particular parlamentaria de un solo partido («espacio libre de coalición"). Pero los coalicionistas se prometieron mutuamente que buscarán un consentimiento en cuestiones importantes. Pero en eso quedó indefinido - sin tener en cuenta un amplio programa de trabajo material previo a la declaración gubernamental- que es una cuestión importante. $Y$ eso debe ser así, porque depende de las peripecias de la política, qué cuestión considera el partido en cierto momento como cuestión importante. Pero según el acuerdo de trabajo, los "problemas de tipo fundamental" deben ser discutidos por la comisión de negociaciones original (una especie de comisión de coalición). De ahí resulta: 
La coalición puede existir solamente mientras que las materias sobre las cuales se puede llegar a un acuerdo, todavía no sean agotadas, y la disposición de ponerse de acuerdo no sea cargada o destruida por la retirada de un coalicionista hacia el espacio libre de coalición y un pacto eventual con otras fuerzas políticas.

En principio, una amplia coalición gubernamental es una buena base para la solución de problemas de gran alcance y con perspectiva a largo plazo, pero tiene un hándicap integrado: no hay ninguna solución «o César, o nada", que también puede ser una ventaja teniendo en cuenta el hecho de que a veces las estimaciones falsas se descubren sólo más tarde y en cuanto a la aceptancia social de decisiones de compromiso. No obstante, parece dificil realizar grandes reformas en el Estado de rendimiento $y$ de distribución del presente que se excedió en el campo financiero $y$ de planificación. Los conflictos de intereses muchas veces sólo pueden ser arreglados cuando la crisis o la dificultad a vencer son de bastante gravedad e impresionabilidad. Se podrían lograr un pensamiento a largo plazo y decisiones que se pueden responder a largo plazo, a cargo de la táctica partidista a corto plazo y ventajas de grupos, sólo por medio de una nueva ética política. Pero ya la conservación de la disposición a negociar y a hacer compromisos es una virtud irrenunciable de una democracia madura. 